\title{
AN OVERVIEW ON DATA MINING DESIGNED FOR IMBALANCED
}

\section{DATASETS}

\author{
Mohammad Imran ${ }^{1}$, Ahmed Abdul Moiz Qyser ${ }^{2}$, Syed Salman Ali ${ }^{3}$, Vijay Kumar.A ${ }^{4}$ \\ ${ }^{1}$ Assistant Professor in CSE, Muffakham Jah College of Engineering and Technology, Telangana, India \\ ${ }^{2}$ Professor in CSE, Muffakham Jah College of Engineering and Technology, Telangana, India \\ ${ }^{3}$ Assistant Professor in CSE, Muffakham Jah College of Engineering and Technology, Telangana, India \\ ${ }^{4}$ Associate Professor in IT, Nallam Malla Reddy Engineering College, Hyderabad, Telangana, India
}

\begin{abstract}
The imbalanced datasets with the classifying categories are not around equally characterized. A problem in imbalanced dataset occurs in categorization, where the amount of illustration of single class will be greatly lesser than the illustrations of the previous classes. Current existence brought improved awareness during implementation of machine learning methods to complex real world exertion, which is considered by several through imbalanced data. In machine learning the imbalanced datasets has become a critical problem and also usually found in many implementation such as detection of fraudulent calls, bio-medical, engineering, remote-sensing, computer society and manufacturing industries. In order to overcome the problems several approaches have been proposed. In this paper a study on Imbalanced dataset problem and examine various sampling method utilized in favour of evaluation of the datasets, moreover the interpretation methods are further suitable for imbalanced datasets mining.
\end{abstract}

Keywords: Imbalance Problems, Imbalanced datasets, sampling strategies, Machine Learning.

$* * *$

\section{INTRODUCTION}

A problem with imbalance in the class sharing got to be more noticeable by means of the implementation through machine learning calculations towards this present reality. As the implementations vary as of information transfers administration [1], bioinformatics [2], text classification [3, 4] speech recognition [5], to exposure spills of oil images through satellite [6]. An imbalance of class distribution can be artifact and/or dissimilar expenses of examples or errors. These have external concentration from Data Mining and machine learning society in variety of Workshops [7] and Special issue [8]. The blend of documents in these venues displayed, determined and everywhere the nature of the class inequality issue confronted through Data Mining society. Inspecting strategies keep up to be generally preferred in the examination work. All things considered the exploration keeps on evolving with unique implementation, as every application gives a compelling issue. The beginning workshops of Solitary center were mainly the execution appraisal criteria used for imbalanced datasets mining. The persuading inquiry, specified dissimilar class disseminations will be: What is a precise appropriation for a calculation of learning? Provost and Weiss reachable a definite examination on the impact of allocation class on learning classifier [9]. Our clarification agrees through their effort that the characteristic dissemination is regularly not the best circulation for taking in a classifier [8]. Likewise, the information with imbalance could be further normal for "sparseness" in class than the peculiarity space than the class awkwardness. Diverse resampling procedures have been utilized, for example, self-assertive oversampling with substitution, self-assertive under testing, persistent oversampling, persistent under inspecting, oversampling with manufactured era of new examples focused around the known data, and mixes of the above methods [8]. Notwithstanding the issue of between class circulation, an alternate vital issue emerging because of the sparsity in information is the conveyance of information inside each one class [7]. This issue was additionally connected to the problem with disjuncts of little during choice of hierarchy knowledge. However an alternate, order of attention is an affirmation based approach as a solitary learner of class. The learners of single class give an alluring substitute towards conventional discriminative move, where in the classifier are taught going on the objective class only.

Here in this composition, we display a moderate diagram issue of imbalanced datasets mining by method for specific concentrate on execution system and inspecting strategies.

\section{PROBLEMS IN IMBALANCE}

The imbalance class issue has gotten huge consideration in ranges, for example, Pattern Recognition and Machine Learning as of late. Multiple class information set is understood as imbalanced, as soon as the classes among a minority is vigorously under spoken towards as opposed to alternate class in the greater part one. This worry is principally key in certifiable usage as it is immoderate to the cases of misclassify from the class of minority, for instance, identification of fraudulent telephone calls, diagnosis of rare diseases, information retrieval, text categorization and filtering tasks [10]. Several perspectives have been earlier anticipated to deal through this trouble, which can be categorized into two groups: 1 . Create innovative algorithms 
otherwise change prevailing ones to obtain the problem of imbalance class into concern is identified as the internal approaches and 2. Data to be pre-process in order to reduce the consequence originate by imbalance class is recognized as exterior approaches. The intramural proposal have the drawback of being algorithm explicit, whereas external approaches are independent of the classifier used and are, more flexible. For this reason in order to solving imbalanced classification problems $\mathrm{CO}^{2} \mathrm{RBFN}$ is applied [11]. The main implementation of the exact classification with class of minority is further important than class of majority. For instance, in predicting protein relations, the numbers of proteins no interacting is greater than number of proteins interacting. Also in medical investigation crisis, the numbers of cases with disease are frequently minor than cases of nondisease [12]. A high activity of advancement in the imbalanced learning problem remains well-informed of all existing developments and will be a difficult assignment. The capacity of data imbalanced to considerably compromise the presentation of most benchmark learning algorithm is the fundamental issue with the imbalanced learning problem. The imbalanced dataset problem occurs in different kinds of fields. In order to highlight the implications of the imbalanced learning problem, this paper presents some of the fields such as, medical diagnosis, text classification, detection of oil spill in radar images, information retrieval that had problems on imbalanced dataset that are represented in figure.

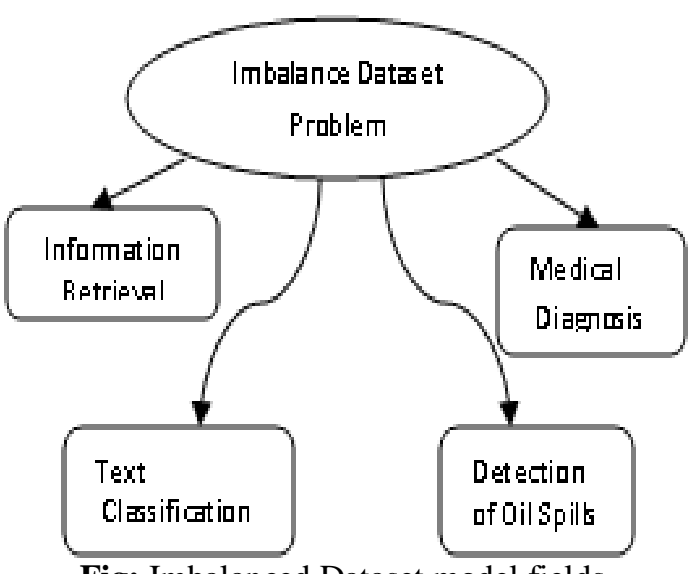

Fig: Imbalanced Dataset model fields.

\section{SAMPLING STRATEGIES}

Sampling Methods proposed by Jong Myong Choi, is an iterative sampling methodology that was used to produce smaller learning sets by removing unnecessary instances. It integrates informative and the representative under-sampling mechanisms to speed up the learning procedure for imbalanced data learning with a SVM. For large-scale imbalanced datasets, sampling methodology provides a resourceful and effective solution for imbalanced data learning with an SVM [13].

Adaptive methods of sampling and generation of data in synthetic, the intention is to provide a distribution of balanced from over-sampling and/or under-sampling methods to improve overall classification. Examining with sampling of synthetic, the SMOTE (Synthetic Minority Over-sampling Methods) made data of synthetic in class with minority by choosing percentage of the closest minority neighbors of a minority data and creating synthetic minority data along with the lines between the minority data and the closest minority neighbors. Versatile sampling methods were planned to generate data of synthetic. The thought of Borderline-SMOTE method was to find out the borderline minority samples. Then, synthetic samples were generated along the line between the marginal samples and their nearest neighbors of the same class [14].

\section{COST-SENSITIVE MEASURES}

\subsection{Cost Matrix}

The cost sensitive process normally expect that the expenses of building a blunder are acknowledged [15]. That is one has an expense lattice, which characterizes the expenses caused in positives with false and negatives with false. Every case, ' $x$ ' could be connected through an expense $c(i, j, x)$, as it characterizes the expense of foreseeing $i$ class used for $x$ when the "genuine" class is $\mathrm{j}$. The objective is to take a choice to reduce the normal expense. The ideal forecast for $\mathrm{x}$ could be characterized like

$$
\sum_{\mathrm{j}} \mathrm{P}(\mathrm{j} \mid \mathrm{x}) \mathrm{C}\left(\mathrm{i}_{,} \mathrm{j}, \mathrm{x}\right)
$$

The previously stated comparison obliges a processing of restrictive probabilities of $\mathrm{j}$ class given peculiarity illustration or vector $\mathrm{x}$. As the expense comparison is clear, we don't generally have an expense joined to making a blunder. The expenses could be diverse for each sample and not just for each sort of lapse. In this manner, $C(i, j)$ is not generally $=$ to $C(i, j, x)$.

\subsection{Cost Curves}

[16] It suggests the cost curves, everywhere an $\mathrm{x}$-axis constitutes to the part of the class with positive in the preparation set, and the y-axis speaks to the normal lapse rate developed on all of the preparation sets. The preparation sets for an information set is created by under (or over) testing. The lapse rates for class circulations not spoke to are interpreted by introduction. They characterize two expense delicate segments for a machine learning calculation: 1) creating a mixed bag of classifiers appropriate for diverse disseminations and 2) Choosing the suitable classifier for the exact appropriation. Then again, as the misclassification expenses are acknowledged, the x-pivot can speak to the "likelihood expense capacity", which is the standardized result of $\mathrm{c}(-\mathrm{I}+) * \mathrm{p}(+)$ the $\mathrm{y}$-axis speaks to the normal expense.

\section{METHODS OF ENSEMBLE BASED}

Blending of classifiers might be a powerful method for enhancing forecast precision. As a standout amongst the most prominent consolidating strategies, boosting [17], utilizes versatile examining of examples to produce a very 
precise group of classifiers whose individual worldwide precision is just direct. In boosting, the classifiers in the gathering are prepared serially, with the weights on the preparation occasions balanced adaptively as indicated by the execution of the past classifiers. The principle thought is that the order calculation ought to focus on the occurrences that are hard to learn.

Not with standing boosting, well known inspecting routines have additionally been conveyed to develop groups. Radivojac et al. [18] joined stowing with systems of oversampling for the bioinformatics area. Liu et al. [19] likewise connected a variation of stowing by bootstrapping at equivalent extents since together the classes of majority and minority. As they implement these methods to the difficulty of sentence border line detection. [20] Phua et. a1 combine bagging and stacking to recognize the greatest blend of classifiers. In their protection misrepresentation discovery area, they record that stacking-sacking accomplishes the preeminent cost-investment funds.

\subsection{SMOTE Boost}

SMOTE Boost algorithm join SMOTE and the principle boosting methodology [21]. We need to use SMOTE for enhancing the exactness above the classes of minority, and to keep up precision over the whole information set we need to use boosting. A real objective is an enhanced model for class of minority in the information set, by giving the learner not just among the class of minority class occurrences that were rnisclassified in past boosting emphases, additionally with a more extensive representation of those occasions. Our objective is to lessen the inclination inborn in the learning methodology because of the class awkwardness, and expand the inspecting weights for the minority class. Presenting SMOTE in every round of boosting would empower every learner to have the capacity to example a greater amount of the minority class cases, furthermore learn better and more extensive choice areas for the minority class.

\section{CONCLUSION}

Mining from imbalanced datasets is in fact an extremely critical issue from mutually with execution and algorithmic viewpoint. Not picking the right appropriation or the destination capacity while creating a classification model can present inclination towards popular share (possibly uninteresting) class. Moreover, prescient precision is not a valuable assess when assessing classifiers adapted on imbalance information sets.

We conclude that, the solution for solving the imbalanced dataset problem is the data level process. Because, the data level process provides better results by using the oversampling algorithm for pre-processing and for balancing we use several algorithms that can be mentioned above. Thus this paper might be useful for the researchers to know about the imbalance dataset problems and also its solutions.

\section{REFERENCES}

[1]. Learning Goal Oriented Bayesian Networks for Telecommunications Risk Management. Singh, M., Ezawa, K., J., and Norton, S., W. (1996). In Transactions of a Machine Learning International Conference, pages 139-147. [2]. Classification and Knowledge Discovery in Protein Databases. (2004) Dunker, K., Chawla, N. V., Radivojac, P., and Obradovic, Z.. Biomedical Informatics Journal, 224$239,37(4)$.

[3]. Heterogeneous Uncertainity Sampling for Supervised Learning. Catlett, J. and Lewis, D. (1994). In Transactions of a Machine Learning $11^{\text {th }}$ International Conference, pages 148-156.

[4]. Inductive Learning Algorithms and Representations for Text Categorization. Platt, J., Dumais, S., Heckerman, D., and Sahami, M. (1998). In Transactions of a Information and Knowledge Management $7^{\text {th }}$ International Conference, pages $148-155$.

[5]. "A Case Study in Machine Learning from Imbalanced Data" for Spoken Language Processing. (2004) Stolcke, Shriberg, Liu, Y., E., A., Chawla, N. V., and Harper, M..

[6]. Machine Learning for the Detection of Oil Spills in Satellite Radar Images. M., Holte, R., Kubat, and Matwin, S. (1998). Machine Learning, 30:195-215.

[7]. A Comparison of Various Strategies : Learning from Imbalanced Data sets. (2000b) Japkowicz, N. In Transactions of the AAA1'2000 Workshop on Learning from Imbalanced Data Sets, Austin, TX.

[8]. SIGKDD Special Issue on Learning from Imbalanced Datasets. Japkowicz, N., Chawla, N. V., and Kokz, A., editors (2004a).

[9]. Learning when Training Data are Costly: The Effect of Class Distribution on Tree Induction. Provost, F. and Weiss, G. (2003). Journal of Artificial Intelligence Research, 19:3 15-354.

[10]. "The class imbalance problem in pattern classification and learning", R.A. Mollineda, R. Alejo, V. García, J.S. Sánchez, J.M. Sotoca, Pattern Analysis and Learning Group, Dept.de Llenguatjes i Sistemes Informàtics, Universitat Jaume I.

[11]. "Analysis of an evolutionary RBFN design algorithm, CO2RBFN, for imbalanced data sets", Alberto Fernández, María Dolores Pérez-Godoy, Antonio Jesús Rivera, María José del Jesus, Pattern Recognition Letters 31 (2010) 23752388.

[12]. "Handling imbalanced data sets with synthetic boundary data generation using bootstrap re-sampling and AdaBoost methodss", Chidchanok Lursinsap, Putthiporn Thanathamathee , Pattern Recognition Letters 34 (2013) 1339-1347.

[13]. " A Selective Sampling Method for Imbalanced Data Learning on Support Vector Machines", Jong Myong Choi ,Iowa State University, cjm7331@gmail.com.

[14]. "A multiple resampling method for learning from imbalanced data sets", Estabrooks, A., Jo, T., Japkowicz, N., Computational Intelligence 20, 18-36, 2004.

[15]. A General Method for Making Classifiers Costsensitive. Domingos, P. (1999). Metacost: In Transactions of the Fifrh ACM SIGKDD International Conference on 
Knowledge Discovery and Data Mining, pages 155-164, San Diego, CA. ACM Press.

[16]. Explicitly Representing Expected Cost: An Alternative to ROC Representation. In Transactions of Knowledge Discovery and Data Mining $6^{\text {th }}$ ACM SIGKDD International Conference, ,Holte, R. C. and Drummond, C. (2000)., pages 198-207, Boston. ACM.

[17]. Experiments with a New Boosting Algorithm. Schapire, R. and Freund, Y. (1996). In $13^{\text {th }}$ International Conference on Machine Learning, Bari, Italy.

[18]. Classification and Knowledge Discovery in Protein Databases. (2004) Dunker, K., Chawla, N. V., Radivojac, P., and Obradovic, Z.. Biomedical Informatics journal, 224-239, 37(4).

[19]. Resampling Methodss for Sentence Boundary Detection: (2004) Stolcke, A., N.V. Chawla, E. Shriberg, Liu, Y., and Harper, M.. "A Case Study in Machine Learning from Imbalanced Data for Spoken Language Processing".

[20]. Alahakoon, D. and Phua, C. (2004). Minority report in fraud detection: Classification of skewed data. SIGKDD Explorations, 6(1).

[21]. Smoteboost: Improving Prediction of the Minority Class in Boosting. (2003b) Bowyer, K ,W, Hall, L. O., Chawla, N. V., and. Lazarevic, A. "Principles and Practice of Knowledge Discovery in Databases", in $7^{\text {th }}$ European Conference. Pages 107-1 19, Dubrovnik, Croatia.

\section{BIOGRAPHIES}

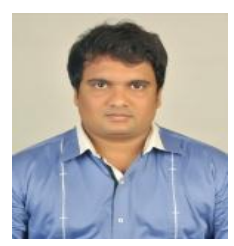

Mohammad Imran ${ }^{1}$ received his B.Tech (CSE) from JNTU, Hyderabad, and M.Tech (CSE) in 2008 from JNTU, Hyderabad, His Research interests include Class Imbalance Learning, Ensemble learning, Machine Learning,Artificial Intelligence and Data Mining. He is currently working as an Assistant Professor in Department of CSE, Muffakham Jah College of Engineering and Technology, Banjara Hills, Hyderabad-500034,India. You can reach him at imran.quba@gmail.com ,mohd.imran@mjcollege.ac.in .

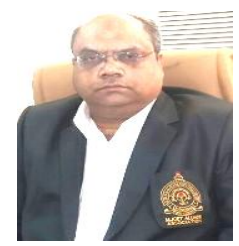

Dr. Ahmed Abdul Moiz Qyser ${ }^{2}$ received his B.E. (Computer Science) in 1994 from Osmania University, M.Tech. (Software Engineering) in 1999 from Jawaharlal Nehru Technological University, Hyderabad, and Ph.D. from Osmania University. His research focus is Software Process Models,Metrics for SMEs and Data Mining. He is presently working as Professor \& Head in Department of Computer Science \& Engineering, Muffakham Jah College of Engineering and Technology, Banjara Hills, Hyderabad-34, India. He is also a visiting Professor to the industry where he teaches Software Engineering and its related areas. He is the author of several research papers in the area of Software Engineering. He is an active member of ACM, CSI and ISTE. You can reach him at aamoiz@gmail.com, aamoiz@mjcollege .ac.in .

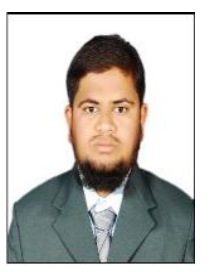

Sd Salman Ali ${ }^{3}$ working as an Assistant Professor in Muffakham Jah College of Engineering and Technology. He completed M.Tech from University College of Engineering, JNTU Anantapur and B.Tech from Jawaharlal Nehru Technological University, Hyderabad. His research areas includes MANETs, Databases, Network Security and Data Mining. You can reach him at ssali.508@gmail.com, salman.ali@mjcollege.ac.in.

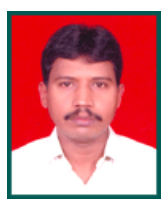

A.Vijaya Kumar completed his M.Tech from Sathyabama University in 2007. Presently he is working as an Associate Professor in the IT Dept in Nalla Malla Reddy Engg. College, Hyderabad. He has published research papers in various National, International conferences, proceedings and Journals. He is a life member of various professional societies like MCSI, MISTE. 